NBI-HE -96-30

ITP-UH-08/96

hep-th/9606004

\title{
Higher genus correlators for the hermitian matrix model with multiple cuts
}

\author{
G. Akemann \\ Niels Bohr Institute \\ Blegdamsvej 17, DK-2100 Copenhagen Ø, Denmark \\ akemann@nbi.dk \\ and \\ Institut für Theoretische Physik, Universität Hannover \\ Appelstraße 2, 30167 Hannover, Germany \\ akemann@itp.uni-hannover.de
}

\begin{abstract}
An iterative scheme is set up for solving the loop equation of the hermitian one-matrix model with a multi-cut structure. Explicit results are presented for genus one for an arbitrary but finite number of cuts. Due to the complicated form of the boundary conditions, the loop correlators now contain elliptic integrals. This demonstrates the existence of new universality classes for the hermitian matrix model. The two-cut solution is investigated in more detail, including the double-scaling limit. It is shown, that in special cases it differs from the known continuum solution with one cut.
\end{abstract}




\section{Introduction}

The study of multi-cut solutions of matrix models is interesting for several reasons. First of all it completes the perturbative analysis around $N=\infty$, exhausting the full parameter space of the coupling constants for all possible kinds of solutions. This is important in order to tell, whether there exist different types of phase transitions, universality classes or continuum limits than the ones, which are already known for the one-cut solution of the model.

Secondly there may be applications apart from quantum gravity to two dimensional QCD or statistical physics. Results of random matrix theory are used for example in the study of the spectrum of the QCD inspired discretised Dirac operator. The merging of several cuts into a one-cut solution of a complex matrix model with "temperature" would be of interest there in various limits (《1] and references therein).

In this paper the approach of iteratively solving the loop equation for the hermitian one-matrix model [2] is chosen and generalised to an arbitrary but finite number of cuts. The success of this method is based on a nonperturbative treatment in the coupling constants, which is valid for an arbitrary polynomial potential. Even away from the double scaling limit higher orders in the genus expansion can be obtained explicitly. The validity for an arbitrary potential makes it possible to read off immediately, whether the results are universal or not. Here this will lead to a whole set of new universality classes for the planar two-point function or two-loop correlator.

Another reason for choosing the loop equation techniques is that it seems, that the method of orthogonal polynomials is not applicable to multi-cut solutions in general. Solving the string equation for the norms of the polynomials numerically, instabilities or a so-called chaotic behaviour has been found by a variety of authors [3, 4, 5, 6, 7]. The region, where these instabilities occur, seems to match precisely with the part of the phase space, where the multi-cut solutions dominate. This has been shown for the hermitian model with an even sextic potential as an example [4, 8]. So apart from the symmetric two-cut solution and some cases of degenerate minima of the potential, where smooth numerical solutions have been found [3, 6, 8], an analytic expression for the orthogonal polynomials even for the planar limit is still missing in the case of a generic multi-cut situation?. As an example

\footnotetext{
${ }^{1}$ The two-component ansatz for the recursion coefficients made in [9, 10 and 11 is restricted to the purely symmetric case of two cuts. The failure of a more general ansatz for more cuts is studied in [12].
} 
the nonsymmetric two-cut solution, which is presented here most explicitly, explores such a region of chaotic numerical solutions [7].

The paper is divided up into two parts. In the first part the iterative scheme for solving the model with $s$ cuts is presented, including results for genus one. The second part is then devoted to a more detailed study of the two-cut solution, which also contains an analysis of the double-scaling limit.

In section 2 and 3 the main definitions and the loop equations for the hermitian model with multiple cuts are displayed, following closely the notation of [2]. The planar solution is derived in section 4, including the required boundary conditions. Then, in section 5 the iterative scheme for higher genera is presented. Special care has to be taken to the inversion of the loop equation due to the zero modes, which occur for more than one cut. The one-loop correlator of genus one is given for an arbitrary number of cuts.

Turning to the two-cut solution, section 6 contains the full two-point function as well as the free energy of genus one. After a short discussion of general properties in the symmetric limit, section 7 deals with the different possibilities of taking a continuum limit, displaying some new results. Section 8 closes with a final discussion and future prospects.

\section{Basic definitions}

Throughout the paper the same notation as in [2] is used, which is redisplayed here for completeness. The partition function of the hermitian one-matrix model is defined by

$$
Z\left[N,\left\{g_{i}\right\}\right] \equiv e^{N^{2} F\left[N,\left\{g_{i}\right\}\right]} \equiv \int d \phi \exp (-N \operatorname{Tr} V(\phi)),
$$

where the integration is over hermitian $N \times N$ matrices $\phi$. The matrix potential is given by the following power series,

$$
V(\phi) \equiv \sum_{j=1}^{\infty} \frac{g_{j}}{j} \phi^{j} .
$$

A specific potential of finite order can be inspected by setting the extra couplings to zero in the final result. In this way of keeping all the coupling constants $g_{i}$ throughout the calculation, they can be used as sources for expectation values] of the following type,

$$
-m \frac{d}{d g_{m}} F\left[N,\left\{g_{i}\right\}\right]=\frac{1}{N}\left\langle\operatorname{Tr} \phi^{m}\right\rangle, \quad m \in \mathrm{N}_{+} .
$$

\footnotetext{
${ }^{2}$ Averages are defined as usual by $\langle Q(\phi)\rangle=\frac{1}{Z} \int d \phi Q(\phi) \exp (-N \operatorname{Tr} V(\phi))$.
} 
Introducing the loop insertion operator

$$
\frac{d}{d V}(p) \equiv-\sum_{j=1}^{\infty} \frac{j}{p^{j+1}} \frac{d}{d g_{j}},
$$

the generating functional or one-loop average $W(p)$ can thus be obtained from the free energy $F$

$$
\begin{aligned}
W(p) & \equiv \frac{1}{N} \sum_{k=0}^{\infty} \frac{\left\langle\operatorname{Tr} \phi^{k}\right\rangle}{p^{k+1}}=\frac{1}{N}\left\langle\operatorname{Tr} \frac{1}{p-\phi}\right\rangle \\
& =\frac{d}{d V}(p) F+\frac{1}{p} .
\end{aligned}
$$

In the same way all the multi-loop correlators can be derived by applying $\frac{d}{d V}(p)$ to $F($ or to $W(p))$

$$
\begin{aligned}
W\left(p_{1}, \ldots, p_{n}\right) & \equiv N^{n-2}\left\langle\operatorname{Tr} \frac{1}{p_{1}-\phi} \cdots \operatorname{Tr} \frac{1}{p_{n}-\phi}\right\rangle_{c o n n} \\
& =\frac{d}{d V}\left(p_{n}\right) \frac{d}{d V}\left(p_{n-1}\right) \cdots \frac{d}{d V}\left(p_{1}\right) F, \quad n \geq 2 .
\end{aligned}
$$

Here conn refers to the connected part. As the loop correlators and the free energy have the same genus expansion,

$$
\begin{aligned}
F & =\sum_{g=0}^{\infty} \frac{1}{N^{2 g}} F_{g} \\
W\left(p_{1}, \ldots, p_{n}\right) & =\sum_{g=0}^{\infty} \frac{1}{N^{2 g}} W_{g}\left(p_{1}, \ldots, p_{n}\right),
\end{aligned}
$$

eq. (2.6) is valid for each genus $g \geq 0$ separately. Finally the asymptotic behaviour of $W(p)$ for large $p$ can be read off from the definition (2.5)

$$
\lim _{p \rightarrow \pm \infty} W(p) \sim \frac{1}{p}
$$

The r.h.s. does not depend on $N$, so the leading contribution clearly comes from the planar part

$$
\begin{aligned}
\lim _{p \rightarrow \pm \infty} W_{0}(p) & \sim \frac{1}{p} \\
\lim _{p \rightarrow \pm \infty} W_{g}(p) & \sim \mathcal{O}\left(\frac{1}{p^{2}}\right), g \geq 1 .
\end{aligned}
$$


Using this fact the genus expansion of eq. (2.5) reads

$$
W_{g}(p)=\frac{d}{d V}(p) F_{g}, \quad g \geq 1,
$$

so the $p$-dependence of $W_{g}(p)$ is completely absorbed in the total derivative. The last two equations will become important, when the genus expanded loop equation is inverted, which determines $W(p)$ iteratively in genus.

\section{The loop equation}

The derivation of the loop equation for multiple cuts can be performed exactly along the same lines like for the one-cut case, exploiting the invariance of the partition function under a field redefinition $\phi \rightarrow \phi+\epsilon /(p-\phi)$. The result differs only by the contour $\mathcal{C}$ of the complex integral (s. fig. 1)

$$
\oint_{\mathcal{C}} \frac{d \omega}{2 \pi i} \frac{V^{\prime}(\omega)}{p-\omega} W(\omega)=(W(p))^{2}+\frac{1}{N^{2}} \frac{d}{d V}(p) W(p), \quad p \notin \sigma,
$$

where $V^{\prime}(\omega)=\sum_{j} g_{j} \omega^{j-1}$. In the derivation it has been assumed like in the one-cut case (e.g. in [13]), that the density $\rho_{N}(\lambda) \equiv \frac{1}{N}\left\langle\sum_{i}^{N} \delta\left(\lambda-\lambda_{i}\right)\right\rangle$ of the eigenvalues of the matrices $\phi$ has a compact support $\sigma$ in the vicinity of $N=\infty$. Here $\sigma$ consists of an arbitrary but fixed number $s$ of distinct intervals

$$
\sigma \equiv \bigcup_{i=1}^{s}\left[x_{2 i}, x_{2 i-1}\right], \quad x_{1}>x_{2}>\ldots>x_{2 s}
$$

From rewriting $W(p)$ in terms of $\rho_{N}(\lambda)$

$$
W(p)=\int d \lambda \frac{\rho_{N}(\lambda)}{p-\lambda}
$$

it is clear, that when expanded at $N=\infty W(p)$ has $s$ cuts along the real axis on $\sigma$ and is analytic elsewhere. Hence the contour $\mathcal{C}$ in the loop equation (3.1) has to enclose all singularities of $W(\omega)$, but not the point $\omega=p$. It may now well be situated between two cuts, in particular a double-scaling limit can be performed at an internal edge of a cut (s. chap. 7).

Inserting the genus expansion eq. (2.8) into the loop equation (3.1) one gets

$$
\oint_{\mathcal{C}} \frac{d \omega}{2 \pi i} \frac{V^{\prime}(\omega)}{p-\omega} W_{0}(\omega)=\left(W_{0}(p)\right)^{2}
$$




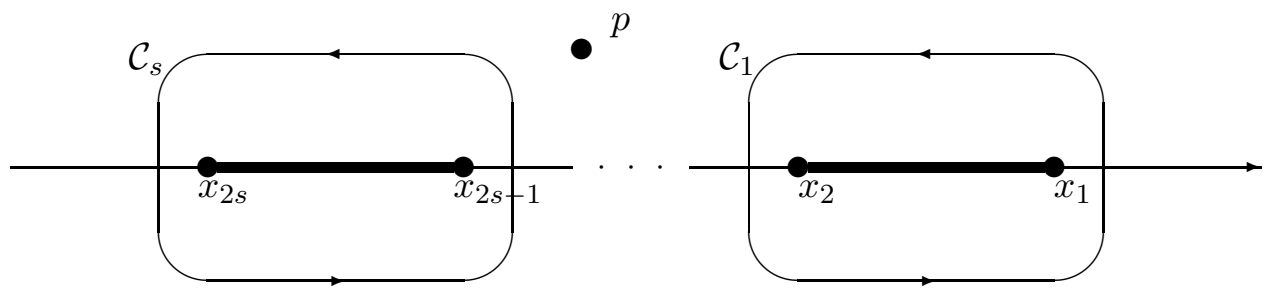

Figure 1: The contour of integration $\mathcal{C}=\cup_{i=1}^{s} \mathcal{C}_{i}$

for genus zero and

$$
\left(\hat{\mathcal{K}}-2 W_{0}(p)\right) W_{g}(p)=\sum_{g \prime=1}^{g-1} W_{g^{\prime}}(p) W_{g-g^{\prime}}(p)+\frac{d}{d V}(p) W_{g-1}(p), \quad g \geq 1,
$$

for higher genera, where $\hat{\mathcal{K}}$ is a linear integral operator defined by

$$
\hat{\mathcal{K}} f(p) \equiv \oint_{\mathcal{C}} \frac{d \omega}{2 \pi i} \frac{V^{\prime}(\omega)}{p-\omega} f(\omega) .
$$

Knowing the result for $W_{0}(p)$ one can determine $W_{g}(p)$ for $g \geq 1$ iteratively from contributions of lower genus on the r.h.s. of eq. (3.5), provided that the operator $\left(\hat{\mathcal{K}}-2 W_{0}(p)\right)$ can be inverted uniquely.

\section{The planar solution}

The quadratic equation (3.4) for the planar solution $W_{0}(p)$ can be solved in the following way. Deforming the contour in eq. (3.4) to infinity one gets

$$
\left(W_{0}(p)\right)^{2}=V^{\prime}(p) W_{0}(p)+\oint_{\mathcal{C}_{\infty}} \frac{d \omega}{2 \pi i} \frac{V^{\prime}(\omega)}{p-\omega} W_{0}(\omega) .
$$

The solution is then formally given by

$$
W_{0}(p)=\frac{1}{2} V^{\prime}(p)-\frac{1}{2} \sqrt{\left(V^{\prime}(p)\right)^{2}+4 Q(p)},
$$




$$
Q(p)=\oint_{\mathcal{C}_{\infty}} \frac{d \omega}{2 \pi i} \frac{V^{\prime}(\omega)}{p-\omega} W_{0}(\omega)
$$

where the minus sign is chosen in order to fulfill the asymptotic eq. (2.10). Now if it is assumed that $W_{0}(p)$ has $s$ cuts in the complex plane, one can make the following ansatz for the square root,

$$
W_{0}(p)=\frac{1}{2}\left(V^{\prime}(p)-M(p) \sqrt{\prod_{i=1}^{2 s}\left(p-x_{i}\right)}\right) .
$$

$M(p)$ is assumed to be an analytic function, which still has to be determined. The signs of the complex square root are defined by the following choice,

$$
\lim _{p \rightarrow \pm \infty} \sqrt{\prod_{i=1}^{2 s}\left(p-x_{i}\right)} \sim p^{s} .
$$

The analyticity of $M(p)$ allows one to write it as

$$
M(p)=\oint_{\mathcal{C}_{\infty}} \frac{d \omega}{2 \pi i} \frac{M(\omega)}{\omega-p} .
$$

Now solving eq. (4.3) for $M(\omega)$ and plugging it into eq. (4.5) yields

$$
M(p)=\oint_{\mathcal{C}_{\infty}} \frac{d \omega}{2 \pi i} \frac{V^{\prime}(\omega)}{(\omega-p) \sqrt{\prod_{i=1}^{2 s}\left(\omega-x_{i}\right)}},
$$

because the $W_{0}(\omega)$-term vanishes due to its asymptotic behaviour at infinity $\sim \frac{1}{\omega}$. When evaluated for a given potential of finite degree $d, M(p)$ will be a polynomial of degree $d-s-1$. Therefore the maximal possible number of cuts has to be $s_{\max } \leq d-1$ ㅇ. Reinserting the solution for $M(p)$ into eq. (4.3) and deforming back the contour leads to the final result, the planar one-loop correlator with an $s$-cut structure,

$$
W_{0}(p)=\frac{1}{2} \oint_{\mathcal{C}} \frac{d \omega}{2 \pi i} \frac{V^{\prime}(\omega)}{p-\omega} \sqrt{\prod_{i=1}^{2 s}\left(\frac{p-x_{i}}{\omega-x_{i}}\right)} .
$$

From this result the planar eigenvalue density $\rho(\lambda) \equiv \lim _{N \rightarrow \infty} \rho_{N}(\lambda)$ can be recovered, taking the limit $N \rightarrow \infty$ of eq. (3.3)

$$
\begin{aligned}
\rho(\lambda) & =\frac{1}{2 \pi i} \lim _{\epsilon \rightarrow 0}\left(W_{0}(\lambda-i \epsilon)-W_{0}(\lambda+i \epsilon)\right) \\
& =\frac{1}{2 \pi}|M(\lambda)| \sqrt{-\prod_{i=1}^{2 s}\left(\lambda-x_{i}\right)}, \quad \lambda \in \sigma .
\end{aligned}
$$

\footnotetext{
${ }^{3}$ It can be shown, that even $s_{\max } \leq \frac{d}{2}$ holds.
} 
Equivalently $\rho(\lambda)$ could have been obtained from the saddle point equation for the partition function in terms of the eigenvalues (see for example in [12]). Here the solution takes a closed form for an arbitrary number of cuts $s$.

In the planar solution for $W_{0}(p)$ eq. (4.7) the edges of the cuts $x_{i}$, $i=1, \ldots, 2 s$, still have to be determined as functions of the coupling constants $g_{i}, i \in N_{+}$. These boundary conditions are derived by exploiting again the asymptotic behaviour of $W_{0}(p) \sim \frac{1}{p}$. All the coefficients in eq. (4.7) of the order $p^{s-1}$ down to $p^{0}$ must vanish, whereas the $\frac{1}{p}$-coefficient must equal unity,

$$
\delta_{k, s}=\frac{1}{2} \oint_{\mathcal{C}} \frac{d \omega}{2 \pi i} \frac{\omega^{k} V^{\prime}(\omega)}{\sqrt{\prod_{i=1}^{2 s}\left(\omega-x_{i}\right)}}, \quad k=0, \ldots, s .
$$

These conditions provide only $s+1$ equations for the $2 s$ constants $x_{i}$. This is sufficient if and only if $s=1$. For $s \geq 2$ the remaining equations are given by a criterion of stability against tunnelling of eigenvalues between different cuts in the limit $N \rightarrow \infty$ [14]. Stability is achieved by imposing equality of the chemical potentials of all cuts in the saddle point equation for the density $\rho(\lambda)$. These conditions can be most suitably written in the form [15]

$$
0=\int_{x_{2 k+1}}^{x_{2 k}} d \lambda M(\lambda) \sqrt{\prod_{i=1}^{2 s}\left(\lambda-x_{i}\right)}, \quad k=1, \ldots, s-1,
$$

where the integration is performed between each two neighbouring cuts. This second type of boundary condition for $s \geq 2$ leads to the appearance of elliptic integrals in the higher genus correlators as well as in the planar multi-loop correlators.

\section{The iterative procedure}

After having solved the planar part of $W(p)$ as a starting point, higher genus contributions can now be iteratively determined by inverting the genus expanded loop equation (3.5). From $W_{g}(p)$ all multi-loop correlators of the same genus can be obtained then by simply applying the loop insertion operator $\frac{d}{d V}(p)$ to it (see eq. (2.6) and (2.5)). Along the same lines like in the one-cut solution [2] a change of variables from coupling constants to moments $M_{i}^{(k)}$ will allow to express the higher genus correlators nonperturbatively in 
the coupling constants $g_{i}$, depending then only on a finite number of these moments,

$$
M_{i}^{(k)} \equiv \oint_{\mathcal{C}} \frac{d \omega}{2 \pi i} V^{\prime}(\omega) \phi_{i}^{(k)}(\omega), \quad k \in \mathrm{N}_{+}, \quad i=1, \ldots, 2 s
$$

with

$$
\phi_{i}^{(k)}(\omega) \equiv \frac{1}{\left(\omega-x_{i}\right)^{k}} \phi^{(0)}(\omega), \quad \phi^{(0)}(\omega) \equiv \frac{1}{\sqrt{\prod_{i=1}^{2 s}\left(\omega-x_{i}\right)}} .
$$

Deforming the contour to infinity and expanding $\phi_{i}^{(k)}(\omega)$ the moments can be seen to depend in the following way on the couplings,

$$
M_{i}^{(k)}=g_{k+s}+g_{k+s+1} \sum_{j=1}^{2 s} x_{j}\left(\frac{1}{2}+k \delta_{i j}\right)+\ldots .
$$

Here an explicit dependence on the number of cuts $s$ enters. The moments may also be used to characterise multi-critical points. Namely because of

$$
M_{i}^{(k)}=\left.\frac{1}{(k-1) !} \frac{d^{k-1}}{d \lambda^{k-1}} M(\lambda)\right|_{\lambda=x_{i}},
$$

the $m$-th multi-critical point at $x_{i}$ is given by

$$
\begin{aligned}
& M_{i}^{(k)}=0, \quad k=1, \ldots, m-1, \\
& M_{i}^{(m)} \neq 0,
\end{aligned}
$$

which indicates that the eigenvalue density $\rho(\lambda)$ in eq. (4.8) accumulates $m-1$ extra zeros at $x_{i}$.

Secondly it can be seen from eqs. (5.4) and (4.3), that the planar oneloop correlator depends on all moments, when $M(\lambda)$ is expanded in a Taylor series. This is a special feature of genus $g=0$ only.

\subsection{Determination of the basis}

In order to proceed it is necessary to introduce a basis for the operator acting on $W_{g}(p)$ in eq. (3.5),

$$
\left(\hat{\mathcal{K}}-2 W_{0}(p)\right) \chi_{i}^{(n)}(p) \equiv \frac{1}{\left(p-x_{i}\right)^{n}}, \quad n \in \mathrm{N}_{+}, i=1, \ldots, 2 s .
$$


Given that the r.h.s. of eq. (3.5) is a fractional rational function of $p$ having poles at the $x_{i}$ only, $W_{g}(p)$ will then have the following structure

$$
W_{g}(p)=\sum_{n=1}^{3 g-1} \sum_{i=1}^{2 s} A_{i, g}^{(n)} \chi_{i}^{(n)}(p), \quad g \geq 1 .
$$

The $A_{i, g}^{(n)}$ are complicated functions of the $x_{i}$ and the moments $M_{i}^{(k)}$. As the order of the highest pole in $W_{g}(p)$ is not changed by assuming a multi-cut structure, $W_{g}(p)$ will depend on at most $2 s(3 g-1)$ moments, following the same arguments like in the one-cut solution [2].

A set of basis functions fulfilling eq. (5.6) is defined by

$\tilde{\chi}_{i}^{(n)}(p) \equiv \frac{1}{M_{i}^{(1)}}\left(\phi_{i}^{(n)}(p)-\sum_{k=1}^{n-1} M_{i}^{(n-k+1)} \tilde{\chi}_{i}^{(k)}(p)\right), n \in \mathrm{N}_{+}, i=1, \ldots, 2 s$,

which can be proven by induction. However, this definition is not unique, as the kernel of $\left(\hat{\mathcal{K}}-2 W_{0}(p)\right)$ is not empty,

$$
\operatorname{Ker}\left(\hat{\mathcal{K}}-2 W_{0}(p)\right)=\operatorname{Span}\left\{p^{l} \phi^{(0)}(p) ; l=0, \ldots, s\right\} .
$$

This can be shown by using the boundary conditions of the first type eq. (4.9). Now because of the asymptotic behaviour eq. (2.10) only terms $\sim \mathcal{O}\left(\frac{1}{p^{2}}\right)$ at large $p$ may be added to $W_{g}(p)$ for $g \geq 1$. This requirement reduces the set of zero modes to be

$$
\text { allowed zero modes : }\left\{p^{l} \phi^{(0)}(p) ; l=0, \ldots, s-2\right\} \text {. }
$$

In particular for the one-cut solution this argument excluded any zero mode to be added, making the definition of the basis in eq. (5.8) unique. In the general case any linear combination of the $s-1$ functions can in principle be added to $W_{g}(p)$ for a solution with $s$ cuts.

In addition to its asymptotic $W_{g}(p)$ has to fulfill eq. (2.11), which can be used to fix the basis uniquely. It says that the $p$-dependence of $W_{g}(p)$ must be completely absorbable into derivatives with respect to $\frac{d}{d V}(p)$. This will only be possible, if the basis functions $\chi_{i}^{(n)}(p)$ may be expressed completely in terms of $\frac{d x_{i}}{d V}(p)$ and $\frac{d M_{i}^{(k)}}{d V}(p)$ as functions of $p$. So the $\tilde{\chi}_{i}^{(n)}(p)$ in eq. (5.8) must be redefined in order to achieve this, which will precisely fix its zero mode content. The derivatives of the $x_{i}$ and the moments $M_{i}^{(k)}$ can be obtained by applying the loop insertion operator $\frac{d}{d V}(p)$ to the definitions 
(5.1) and to the boundary conditions, when rewriting it in the following way:

$$
\begin{aligned}
\frac{d}{d V}(p) & =\frac{\partial}{\partial V}(p)+\sum_{i=1}^{2 s} \frac{d x_{i}}{d V}(p) \frac{\partial}{\partial x_{i}}+\sum_{i=1}^{2 s} \sum_{k=1}^{\infty} \frac{d M_{i}^{(k)}}{d V}(p) \frac{\partial}{\partial M_{i}^{(k)}} \\
\frac{\partial}{\partial V}(p) & \equiv-\sum_{j=1}^{\infty} \frac{j}{p^{j+1}} \frac{\partial}{\partial g_{j}} .
\end{aligned}
$$

Using the identity

$$
\frac{\partial}{\partial V}(p) V^{\prime}(\omega)=\frac{-1}{(p-\omega)^{2}}
$$

the result for the moments reads

$$
\begin{aligned}
\frac{d M_{i}^{(k)}}{d V}(p)= & \left(k+\frac{1}{2}\right)\left(M_{i}^{(k+1)} \frac{d x_{i}}{d V}(p)-\phi_{i}^{(k+1)}(p)\right) \\
& +\frac{1}{2} \sum_{\substack{j=1 \\
j \neq i}}^{2 s} \sum_{l=1}^{k} \frac{1}{\left(x_{j}-x_{i}\right)^{k-l+1}}\left(\phi_{i}^{(l)}(p)-M_{i}^{(l)} \frac{d x_{j}}{d V}(p)\right) \\
& +\frac{1}{2} \sum_{\substack{j=1 \\
j \neq i}}^{2 s} \frac{1}{\left(x_{j}-x_{i}\right)^{k}}\left(M_{j}^{(1)} \frac{d x_{j}}{d V}(p)-\phi_{j}^{(1)}(p)\right), \\
& i=1, \ldots, 2 s, k \in \mathrm{N}_{+} .
\end{aligned}
$$

The quantities $\frac{d x_{i}}{d V}(p)$ are given by the solution of the following set of linear equations,

$$
\begin{aligned}
& 0= \sum_{i=1}^{2 s}\left(x_{i}^{k} M_{i}^{(1)} \frac{d x_{i}}{d V}(p)-p^{k} \phi_{i}^{(1)}(p)\right)+2 k p^{k-1} \phi^{(0)}(p), \quad k=0, \ldots, s, \\
& 0= \sum_{i=1}^{2 s}\left(M_{i}^{(1)} \frac{d x_{i}}{d V}(p)-\phi_{i}^{(1)}(p)\right) K_{i, j}, \quad j=1, \ldots, s-1, \\
& K_{i, j} \equiv \int_{x_{2 j+1}}^{x_{2 j}} d \lambda \frac{\sqrt{\prod_{k=1}^{2 s}\left(\lambda-x_{k}\right)}}{\left(\lambda-x_{i}\right)},
\end{aligned}
$$

where $\frac{d}{d V}(p)$ has been applied to eqs. (4.9) and (4.10). The result of the latter is derived in appendix A, where some care has to be taken to the interchanging of $\frac{d}{d V}(p)$ and deforming the contour in eq. (4.6) for $M(\lambda)$ to 
infinity. It can easily be seen from the linear system of equations (5.14), that the solution will always take the form

$$
M_{i}^{(1)} \frac{d x_{i}}{d V}(p)=\phi_{i}^{(1)}(p)+\sum_{l=0}^{s-2} \alpha_{i, l} p^{l} \phi^{(0)}(p), \quad i=1, \ldots, 2 s
$$

The $\alpha_{i, l}, i=1, \ldots, 2 s, l=0, \ldots, s-2$, will then be determined by the following set of equations:

$$
\begin{aligned}
& 0=\sum_{i=1}^{2 s} \sum_{l=0}^{s-2} \alpha_{i, l} x_{i}^{k} p^{l}-\sum_{i=1}^{2 s} \sum_{l=0}^{k-1} x_{i}^{k-1-l} p^{l}+2 k p^{k-1}, \quad k=0, \ldots, s \\
& 0=\sum_{i=1}^{2 s} \sum_{l=0}^{s-2} \alpha_{i, l} K_{i, j} p^{l}, \quad j=1, \ldots, s-1
\end{aligned}
$$

Comparing the coefficients in powers of $^{l}, l=0, \ldots, s-2$, leads to $2 s(s-1)$ equations for the $\alpha_{i, l}$. They will only depend on the $x_{i}$ and $K_{i, j}$. Hence the only difference between the $\frac{d x_{i}}{d V}(p)$ in eq. (5.15) and the respective quantities in the one-cut solution [2] is the occurrence of a linear combination of the allowed zero modes from eq. (5.10). More explicit results for $s=2$ are given in the next chapter.

Now all necessary ingredients for redefining the basis (5.8) in terms of total derivatives are collected. Solving eq. (5.13) for $\phi_{i}^{(k+1)}(p)$ and expressing its $p$-dependence as $\frac{d}{d V}(p)$-terms plus zero modes, a unique basis can now be obtained inductively from eq. (5.8) by subtracting these corresponding zero modes:

$$
\begin{gathered}
\chi_{i}^{(n)}(p) \equiv \frac{1}{M_{i}^{(1)}}\left(\left.\phi_{i}^{(n)}(p)\right|_{\frac{d}{d V}-p a r t}-\sum_{k=1}^{n-1} M_{i}^{(n-k+1)} \chi_{i}^{(k)}(p)\right), \\
i=1, \ldots, 2 s, n \in \mathrm{N}_{+} .
\end{gathered}
$$

The first basis functions then read

$$
\begin{aligned}
\chi_{i}^{(1)}(p) & =\frac{d x_{i}}{d V}(p), \quad i=1, \ldots, 2 s \\
\chi_{i}^{(2)}(p) & =-\frac{2}{3} \frac{d}{d V}(p) \ln \left|M_{i}^{(1)}\right|-\frac{1}{3} \sum_{\substack{j=1 \\
j \neq i}}^{2 s} \frac{d}{d V}(p) \ln \left|x_{i}-x_{j}\right| .
\end{aligned}
$$

\footnotetext{
${ }^{4}$ The coefficient of $p^{k-1}$ is zero for $k=s$.
} 


\subsection{Calculation of genus 1}

Having determined the basis, the loop equation can now be inverted step by step in genus. For genus $g=1$ eq. (3.5) reads

$$
\left(\hat{\mathcal{K}}-2 W_{0}(p)\right) W_{1}(p)=\frac{d}{d V}(p) W_{0}(p) .
$$

Using the result for $W_{0}(p)$ eq. (4.7) and the loop insertion operator from eq. (5.11) the r.h.s. is given by

$$
\begin{aligned}
\frac{d}{d V}(p) W_{0}(p)= & -\frac{3}{16} \sum_{i=1}^{2 s} \frac{1}{\left(p-x_{i}\right)^{2}}-\frac{1}{8} \sum_{\substack{i, j=1 \\
i<j}}^{2 s} \frac{1}{\left(p-x_{i}\right)\left(p-x_{j}\right)} \\
& +\frac{1}{4} \frac{1}{\phi^{(0)}(p)} \sum_{i=1}^{2 s} \frac{1}{p-x_{i}} M_{i}^{(1)} \frac{d x_{i}}{d V}(p) \\
= & \frac{1}{16} \sum_{i=1}^{2 s} \frac{1}{\left(p-x_{i}\right)^{2}}-\frac{1}{8} \sum_{\substack{i, j=1 \\
i<j}}^{2 s} \frac{1}{x_{i}-x_{j}}\left(\frac{1}{\left(p-x_{i}\right)}-\frac{1}{\left(p-x_{j}\right)}\right) \\
& +\frac{1}{4} \sum_{i=1}^{2 s} \sum_{l=0}^{s-2} \frac{\alpha_{i, l} x_{i}^{l}}{p-x_{i}} .
\end{aligned}
$$

Here the fact has been used, that all the regular parts coming from $\frac{p^{l}}{p-x_{i}}, l=$ $1, \ldots, s-2$, will vanish due to eq. (5.16), such that $W_{0}(p, p)=\frac{d}{d V}(p) W_{0}(p)$ fulfils its correct asymptotic. The two-loop correlator at different arguments $W_{0}(p, q)$ can be obtained in the same way, which is derived in appendix B. The result for the one-loop correlator of genus one with $s$ cuts can now easily be obtained by using the basis eq. (5.18),

$$
\begin{aligned}
W_{1}(p)= & \frac{1}{16} \sum_{i=1}^{2 s} \chi_{i}^{(2)}(p)-\frac{1}{8} \sum_{\substack{i, j=1 \\
i<j}}^{2 s} \frac{1}{x_{i}-x_{j}}\left(\chi_{i}^{(1)}(p)-\chi_{j}^{(1)}(p)\right) \\
& +\frac{1}{4} \sum_{i=1}^{2 s} \sum_{l=0}^{s-2} \alpha_{i, l} x_{i}^{l} \chi_{i}^{(1)}(p) .
\end{aligned}
$$

The integration of $W_{1}(p)$ in order to get $F_{1}$ as well as the calculation of higher genera gets technically very much involved, as the integrals $K_{i, j}$ then have to be integrated or differentiated with respect to the $x_{i}, i=1, \ldots, 2 s$.

For the case of two cuts, the integrals $K_{i, 1}, i=1, \ldots, 4$, can be expressed by the well known complete elliptic integrals of the first, second and third 
kind, making a more detailed analysis possible. This will be the subject of the next chapter.

\section{The two-cut solution}

The case of two cuts may already appear for the symmetric quartic potential, when the coupling constants are suitably chosen [16]. The approach presented here allows for a closed treatment of an arbitrary polynomial potential including higher genus contributions. It is beyond the scope of the method of orthogonal polynomials, as it has been mentioned already in the introduction.

The explicit solution for the zero mode coefficients $\alpha_{i, 0}$ in eq. (5.15) will allow to inspect the planar two-loop correlator in more detail. Knowing the complete $x_{i}$-dependence of these coefficients makes it also possible to study the double-scaling limit. The set of equations (5.16) reads for $s=2$

$$
\begin{aligned}
& 0=\sum_{i=1}^{4} \alpha_{i} \\
& 0=\sum_{i=1}^{4} \alpha_{i} x_{i}-2 \\
& 0=\sum_{i=1}^{4}\left(\alpha_{i} x_{i}^{2}-x_{i}\right) \\
& 0=\sum_{i=1}^{4} \alpha_{i} K_{i},
\end{aligned}
$$

where $\alpha_{i} \equiv \alpha_{i, 0}$ and

$$
K_{i} \equiv \int_{x_{3}}^{x_{2}} d \lambda \frac{\sqrt{\prod_{j=1}^{4}\left(\lambda-x_{j}\right)}}{\left(\lambda-x_{i}\right)}, \quad i=1, \ldots, 4 .
$$

The solution for the integrals $K_{i}$ in terms of complete elliptic integrals may be taken from [17], the result being displayed in appendix C. The solution of the eqs. (6.1) reads

$$
M_{i}^{(1)} \frac{d x_{i}}{d V}(p)=\phi_{i}^{(1)}(p)+\alpha_{i} \phi^{(0)}(p), \quad i=1, \ldots, 4,
$$


where the $\alpha_{i}$ can be found after a tedious calculation to be

$$
\begin{aligned}
\alpha_{1}= & \frac{1}{x_{1}-x_{4}}\left(1+\frac{\left(x_{2}-x_{4}\right)}{\left(x_{1}-x_{2}\right)} \frac{E(k)}{K(k)}\right), \\
\alpha_{2}= & \frac{1}{x_{2}-x_{3}}\left(1+\frac{\left(x_{1}-x_{3}\right)}{\left(x_{2}-x_{1}\right)} \frac{E(k)}{K(k)}\right), \\
\alpha_{3}= & \frac{1}{x_{3}-x_{2}}\left(1+\frac{\left(x_{2}-x_{4}\right)}{\left(x_{4}-x_{3}\right)} \frac{E(k)}{K(k)}\right), \\
\alpha_{4}= & \frac{1}{x_{4}-x_{1}}\left(1+\frac{\left(x_{1}-x_{3}\right)}{\left(x_{3}-x_{4}\right)} \frac{E(k)}{K(k)}\right), \\
& k^{2}=\frac{\left(x_{1}-x_{4}\right)\left(x_{2}-x_{3}\right)}{\left(x_{1}-x_{3}\right)\left(x_{2}-x_{4}\right)} .
\end{aligned}
$$

Having the explicit result for the $\frac{d x_{i}}{d V}(p)$ at hand, the planar two-loop correlator can be evaluated also for different arguments most explicitly. It is derived in appendix $B$, which reads

$$
\begin{aligned}
W_{0}(p, q)= & \frac{1}{4(p-q)^{2}}\left(\sqrt{\frac{\left(p-x_{1}\right)\left(p-x_{4}\right)\left(q-x_{2}\right)\left(q-x_{3}\right)}{\left(p-x_{2}\right)\left(p-x_{3}\right)\left(q-x_{1}\right)\left(q-x_{4}\right)}}\right. \\
& \left.+\sqrt{\frac{\left(p-x_{2}\right)\left(p-x_{3}\right)\left(q-x_{1}\right)\left(q-x_{4}\right)}{\left(p-x_{1}\right)\left(p-x_{4}\right)\left(q-x_{2}\right)\left(q-x_{3}\right)}}\right) \\
+ & \frac{1}{4} \frac{1}{\sqrt{\prod_{j=1}^{4}\left(p-x_{j}\right)\left(q-x_{j}\right)}} \frac{E(k)}{K(k)} f\left(\left\{x_{i}\right\}\right)-\frac{1}{2(p-q)^{2}}
\end{aligned}
$$

The function $f\left(\left\{x_{i}\right\}\right)$ of the $x_{i}$ is given in eq. (B.6). The result fulfils its required analyticity properties (see appendix B). As it must be regular at coinciding arguments, it has to be compared with eq. (5.20) for the two-cut solution, which can also be found in appendix B,

$$
\begin{aligned}
W_{0}(p, p) & =\frac{1}{16} \sum_{i=1}^{4} \frac{1}{\left(p-x_{i}\right)^{2}}+\frac{1}{4}\left(\frac{1}{\left(p-x_{1}\right)\left(p-x_{4}\right)}+\frac{1}{\left(p-x_{2}\right)\left(p-x_{3}\right)}\right) \\
& -\frac{1}{8} \sum_{i<j}^{4} \frac{1}{\left(p-x_{i}\right)\left(p-x_{j}\right)}+\frac{1}{4} \frac{1}{\prod_{j=1}^{4}\left(p-x_{j}\right)} \frac{E(k)}{K(k)} f\left(\left\{x_{i}\right\}\right) .
\end{aligned}
$$

These results for the planar two-loop correlator are clearly universal, as they depend on the coupling constants only implicitly via the endpoints of the cuts. The part proportional to the elliptic integrals simplifies considerably 
in the limit of a symmetric potential, where $x_{4}=-x_{1}$ and $x_{3}=-x_{2}$. The modulus will then be

$$
k_{\text {sym }}^{2}=\frac{4 x_{1} x_{2}}{\left(x_{1}+x_{2}\right)^{2}},
$$

and the function $f\left(\left\{x_{i}\right\}\right)$ becomes

$$
f_{\text {sym }}\left(\left\{x_{i}\right\}\right)=\left(x_{1}+x_{2}\right)^{2} .
$$

\subsection{Results for genus $g=1$}

With the more explicit results for the two-cut solution at hand the free energy $F_{1}$ can now be integrated from $W_{1}(p)$. As it has been mentioned already the knowledge of $F_{1}$ in not necessary to get the higher orders of $W_{g}(p)$ though. Inserting the basis functions eq. (5.18) into eq. (5.21) $W_{1}(p)$ reads

$$
\begin{aligned}
W_{1}(p)= & -\frac{1}{24} \sum_{i=1}^{4} \frac{d}{d V}(p) \ln \left|M_{i}^{(1)}\right|-\frac{1}{6} \sum_{i<j} \frac{d}{d V}(p) \ln \left|x_{i}-x_{j}\right| \\
& +\frac{1}{4} \sum_{i=1}^{4} \alpha_{i} \frac{d x_{i}}{d V}(p) .
\end{aligned}
$$

Using the well known relation for the integral $K(k)$,

$$
\frac{\partial}{\partial k^{2}} K(k)=\frac{1}{2 k^{2}\left(1-k^{2}\right)}\left(E(k)-\left(1-k^{2}\right) K(k)\right),
$$

the following helpful relation can be derived,

$2 \frac{d}{d V}(p) \ln |K(k)|=-\sum_{i=1}^{4} \alpha_{i} \frac{d x_{i}}{d V}(p)+\frac{d}{d V}(p) \ln \left|x_{1}-x_{3}\right|+\frac{d}{d V}(p) \ln \left|x_{2}-x_{4}\right|$.

The free energy $F_{1}$ now can be read off easily from eq. (2.11), $W_{1}(p)=$ $\frac{d}{d V}(p) F_{1}$,

$$
\begin{aligned}
F_{1}= & -\frac{1}{24} \sum_{i=1}^{4} \ln \left|M_{i}^{(1)}\right|-\frac{1}{2} \ln |K(k)|-\frac{1}{6} \sum_{i<j} \ln \left|x_{i}-x_{j}\right| \\
& +\frac{1}{4}\left(\ln \left|x_{1}-x_{3}\right|+\ln \left|x_{2}-x_{4}\right|\right) .
\end{aligned}
$$


Taking the symmetric limit of genus one of the $O(n)$-model for $n=+2[18]$.

The calculation of higher genera may now be performed by computer algebra, using eq. (3.5). Taking the derivative $\frac{d}{d V}(p)$, all quantities can expressed algebraically by the known expressions for the $\frac{d x_{i}}{d V}(p), \frac{d M_{i}^{(k)}}{d V}(p)$ and $K(k)$ and $E(k)$, using eq. 6.10) and a similar expression for $E(k)$.

The particular case of having two cuts allows for a certain check of the one-loop correlator, which is valid for all genera. If one is taking the symmetric limit at the end of the calculation, which is implemented by setting the odd coupling constants $g_{2 i+1}, i \in \mathrm{N}$, to zero, all the odd powers of expectation values of matrices will vanish,

$$
\left\langle\operatorname{Tr} \phi^{2 k+1}\right\rangle_{s y m}=0 \quad k \in \mathrm{N} .
$$

Consequently $W(p)$ will become an odd function in $p$,

$$
\begin{aligned}
W^{\text {sym }}(p) & =\frac{1}{N}\left\langle\operatorname{Tr} \frac{1}{p-\phi}\right\rangle_{\text {sym }}=\frac{1}{N} \sum_{k=0}^{\infty} \frac{\left\langle\operatorname{Tr} \phi^{2 k}\right\rangle_{s y m}}{p^{2 k+1}} \\
& =\frac{1}{p}+\frac{1}{N} \frac{\left\langle\operatorname{Tr} \phi^{2}\right\rangle_{s y m}}{p^{3}}+\ldots
\end{aligned}
$$

In particular the term $\sim \mathcal{O}\left(\frac{1}{p^{2}}\right)$ will disappear for all genera. Now the only function of $p$ contained in the basis with this asymptotic behaviour is the zero mode $\phi^{(0)}(p)$. Hence it is not contributing to the symmetric one-loop correlator $W_{g}^{\text {sym }}(p)$ for all genera. Using this special property the result for $W_{1}(p)$ can be checked in this limit. Extracting the $\phi^{(0)}(p)$-dependence from eqs. (6.9) and (6.11) it can be shown, that its coefficient indeed vanishes in the symmetric limit.

\footnotetext{
${ }^{5}$ The moments will then behave like $M_{4}^{(n)}=(-)^{n} M_{1}^{(n)}, M_{3}^{(n)}=(-)^{n} M_{2}^{(n)}$.

${ }^{6}$ For $s \geq 3$ there will always be zero modes left with an asymptotic $\sim \mathcal{O}\left(\frac{1}{p^{3}}\right)$.
} 


\section{The double-scaling limit of the two-cut solution}

It has been argued that in the double-scaling limit (d.s.l.) all the multicut solutions should be equivalent to the one-cut solution for the following reason. Adjusting the coupling constants to achieve a multi-critical behaviour at one of the edges of the cuts, say at $x_{j}$, the d.s.l. magnifies the accumulation of zeros at $x_{j}$ such that the structure elsewhere can be neglected. However, this argument is only true, if the cuts do not touch or vanish at the same time. The given solution with two cuts therefore supplies an example, where this can be checked explicitly. It will be verified, that the generic d.s.l. at any $x_{j}$ is equivalent to the d.s.l. of the one-cut solution [2]. But if the scaling limit is taken at a cut, which simultaneously shrinks to zero, or merges with the other cut, or both, a different continuum behaviour will turn out.

\subsection{Scaling limit at $x_{j}$}

In the generic case the d.s.l. is performed at one specific edge of the cuts $x_{j}$, where for the $m$-th multi-critical point $m-1$ extra zeros accumulate in the eigenvalue density eq. (4.8). Fixing the coupling constants appropriately to reach this point, $p$ and $x_{j}$ will scale in the following way

$$
\begin{aligned}
x_{j} & =x_{j}^{c}-a \Lambda^{\frac{1}{m}} \\
p & =x_{j}^{c}+a \pi,
\end{aligned}
$$

whereas the $x_{i \neq j}$ are kept fixed. For an $m$-th multi-critical point the moments scale according to

$$
M_{j}^{(k)} \sim a^{m-k}, k=1, \ldots, m-1,
$$

where again the $M_{i \neq j}^{(k)}$ do not scale. Looking at the explicit solution for the first basis function, which is given by eqs. (6.3) and (6.4), it is clear, that the zero mode contribution will be sub-dominant in the scaling limit eq. (7.1),

$$
\frac{d x_{j}}{d V}(p)=\frac{1}{M_{j}^{(1)}} \phi_{j}^{(1)}(p) \quad(\text { d.s.l. }) .
$$

The derivatives of the moments take the form

$$
\frac{d M_{j}^{(k)}}{d V}(p)=\left(k+\frac{1}{2}\right)\left(M_{j}^{(k+1)} \frac{d x_{j}}{d V}(p)-\phi_{j}^{(k+1)}(p)\right),
$$




$$
\phi_{j}^{(k+1)}(p)=\frac{1}{\left(p-x_{j}\right)^{k+1}} \frac{1}{\sqrt{\left(p-x_{j}\right) \prod_{i \neq j}\left(x_{j}^{c}-x_{i}\right)}} \text { (d.s.l.) }
$$

Looking at the inductive construction of the basis eq. (5.17) it is obvious then, that the zero modes will be also sub-dominant for the rest of the basis, which is hence given by

$$
\begin{aligned}
\chi_{j}^{(n)}(p) & \equiv \frac{1}{M_{j}^{(1)}}\left(\phi_{j}^{(n)}(p)-\sum_{k=1}^{n-1} M_{j}^{(n-k+1)} \chi_{j}^{(k)}(p)\right), n \in \mathrm{N}_{+} \\
& \sim a^{-m-n+\frac{1}{2}} .
\end{aligned}
$$

Consequently in the scaling limit eq. (7.1) the basis for the poles at $x_{j}$ precisely looks like the one for the one-cut case at $x=x_{j}$ [2]. The other basis functions, which are sub-dominant $\sim a^{\frac{1}{2}}$, will not be needed, as only contributions from poles at $x_{j}$ are dominant. The complete equivalence to the one-cut solution in the d.s.l. will now be shown by looking at the starting point of the iteration $W_{0}(p, p)$ and at the scaled loop insertion operator.

It is easy to see from eq. (5.20), that the leading part in orders of $a$ will be

$$
W_{0}(p, p)=\frac{1}{16} \frac{1}{\left(p-x_{j}\right)^{2}} \quad(\text { d.s.l. }),
$$

which is the same like in the one-cut solution. The scaled $\frac{d}{d V}(p)$ from eq. (5.11) will take the form

$$
\frac{d}{d V}(p)=\frac{d x_{j}}{d V}(p) \frac{\partial}{\partial x_{j}}+\sum_{k=1}^{\infty} \frac{d M_{j}^{(k)}}{d V}(p) \frac{\partial}{\partial M_{j}^{(k)}} \quad \text { (d.s.l.) }
$$

where $\frac{d x_{j}}{d V}(p)$ and $\frac{d M_{j}^{(k)}}{d V}(p)$ are given by eq. (7.3) and eq. (7.4) respectively. Hence the generic d.s.l. of the two-cut solution precisely maps to the d.s.l. of the one-cut solution in [2], when the quantities $d_{c}$ and $M_{k}$ there are replaced by $d_{c} \equiv \prod_{i \neq j}\left(x_{j}^{c}-x_{i}\right)$ and $M_{j}^{(k)}$ respectively here. However, one has to bare in mind, that in this symbolical equivalence of the respective formulas the moments will explicitly depend on the number of cuts as functions of the couplings (see eq. (5.3)).

In reference [2] explicit results up to and including genus $g=4$ are presented in the d.s.l., which are valid here as well. The analysis concerning the possible kinds of combinations of moments, that can appear in eq. (5.7), also applies here. 


\subsection{Merging and shrinking cuts in the d.s.l.}

A different scaling behaviour may be expected, when the d.s.l. is performed at a point, where the two cuts merge, or one of them shrinks to zero, or both happens together. When the case of merging is considered, it has to be taken into account, that extra zeros of $M(p)$ lying between the cuts will be picked up in this limit ${ }^{7}$. This will yet only affect the order of multi-criticality, but not the scaling behaviour of the zero modes.

The outcome of the analysis will be, that in the d.s.l. at either merging or shrinking cuts the zero mode contributions will stay sub-dominant, although becoming singular in the latter case. Nevertheless the continuum results will change in all cases, as in eq. (5.20) more terms will contribute. When the shrinking and merging of the cuts is considered simultaneously, say $x_{2}, x_{4} \rightarrow$ $x_{3}$, even the zero modes will be enhanced such that they contribute in the d.s.l., leading to another different continuum theory.

First the situation of the two cuts merging is considered in the limit $x_{2} \rightarrow x_{3}$, which can be parametrised by

$$
\begin{aligned}
& x_{2}=x_{3}^{c}+a \nu, \quad \nu>0, \\
& x_{3}=x_{3}^{c}-a \mu, \quad \mu>0, \\
& p=x_{3}^{c}+a \pi,
\end{aligned}
$$

as at the critical point $p-x_{3}$ will also scale. Looking at the explicit results eq. (6.4) for the zero modes, the modulus $k^{2}$ will be of the order $a$,

$$
k^{2}=a(\nu+\mu) \frac{\left(x_{1}-x_{4}\right)}{\left(x_{1}-x_{3}^{c}\right)\left(x_{3}^{c}-x_{4}\right)}+\mathcal{O}\left(a^{2}\right) .
$$

So the elliptic integrals can be expanded in $k^{2}$, which reads

$$
\frac{E(k)}{K(k)}=1-\frac{1}{2} k^{2}+\mathcal{O}\left(a^{2}\right) .
$$

The scaling behaviour in $\alpha_{2}$ and $\alpha_{3}$ coming from the factor $\frac{1}{x_{2}-x_{3}}$ will thus be cancelled, leaving them sub-dominant as in the generic case,

$$
\alpha_{1}=\frac{1}{x_{1}-x_{3}^{c}}
$$

\footnotetext{
${ }^{7}$ From analyticity properties and the positivity of the eigenvalue density it follows [9], that $M(p)$ will always have at least one real zero between two adjacent cuts for arbitrary $s$.
} 


$$
\begin{aligned}
\alpha_{2} & =\frac{1}{x_{3}^{c}-x_{1}}\left(1-\frac{1}{2} \frac{\left(x_{1}-x_{4}\right)}{\left(x_{3}^{c}-x_{4}\right)}\right), \\
\alpha_{3} & =\frac{1}{x_{3}^{c}-x_{4}}\left(1-\frac{1}{2} \frac{\left(x_{1}-x_{4}\right)}{\left(x_{1}-x_{3}^{c}\right)}\right), \\
\alpha_{4} & =\frac{1}{x_{4}-x_{3}^{c}} .
\end{aligned}
$$

The coefficients $\alpha_{1}$ and $\alpha_{4}$ are regular in the limit eq. (7.8) anyway. The result for the $\frac{d x_{i}}{d V}(p), i=2,3$, will therefore be the same like in eq. (7.3). Still, the continuum limit is altered, as in eq. (5.13) more terms will survive now in comparison to eq. (7.4) in the generic case,

$$
\begin{aligned}
\frac{d M_{2}^{(k)}}{d V}(p) & =\left(k+\frac{1}{2}\right)\left(\frac{M_{2}^{(k+1)}}{M_{2}^{(1)}} \phi_{2}^{(1)}(p)-\phi_{2}^{(k+1)}(p)\right) \\
& +\frac{1}{2} \sum_{l=1}^{k} \frac{1}{\left(x_{3}-x_{2}\right)^{k-l+1}}\left(\phi_{2}^{(l)}(p)-\frac{M_{2}^{(l)}}{M_{3}^{(1)}} \phi_{3}^{(1)}(p)\right)
\end{aligned}
$$

and the same for the indices 3 and 2 interchanged. The d.s.l. performed at the interior of the two cuts merging therefore leads to a new continuum limit of the theory. The starting point for the iterative solution in the scaling limit reads

$$
W_{0}(p, p)=\frac{1}{16} \sum_{i=2,3} \frac{1}{\left(p-x_{i}\right)^{2}}-\frac{1}{8} \frac{1}{x_{2}-x_{3}}\left(\frac{1}{p-x_{2}}-\frac{1}{p-x_{3}}\right) .
$$

Using eqs. (7.3) and (7.12) to obtain the double-scaled basis, the genus one result can easily be achieved.

Second, the case of the scaling limit is considered, which is performed at a cut simultaneously shrinking to zero, say $x_{4} \rightarrow x_{3}$. It is conveniently parametrised by

$$
\begin{aligned}
x_{3} & =x_{3}^{c}+a \nu, \quad \nu>0, \\
x_{4} & =x_{3}^{c}-a \mu, \quad \mu>0, \\
p & =x_{3}^{c}+a \pi,
\end{aligned}
$$

where the other possible case $x_{2} \rightarrow x_{1}$ can be obtained by interchanging the indices $4 \leftrightarrow 1$ and $3 \leftrightarrow 2$ everywhere. The modulus $k^{2}$ will reach unity in the limit (7.14), where the integral of the first kind $K(k)$ becomes singular. The 
integrals in eq. (6.4) can now be expanded in terms of the complementary modulus $k^{\prime 2}$,

$$
k^{\prime 2} \equiv 1-k^{2}=a(\nu+\mu)\left(\frac{1}{x_{2}-x_{3}^{c}}-\frac{1}{x_{1}-x_{3}^{c}}\right)+\mathcal{O}\left(a^{2}\right) .
$$

Consequently the factor $\frac{E(k)}{K(k)}$ vanishes logarithmically,

$$
\frac{E(k)}{K(k)} \sim \frac{1}{\ln a}+\mathcal{O}(a \ln a) .
$$

This again spoils the possible dominance of the zero modes, the result reading

$$
\begin{aligned}
\alpha_{1} & =\frac{1}{x_{1}-x_{3}^{c}}, \\
\alpha_{2} & =\frac{1}{x_{2}-x_{3}^{c}}, \\
\alpha_{3}, \alpha_{4} & \sim \frac{1}{a \ln a} .
\end{aligned}
$$

So although becoming singular in the limit (7.14) the zero mode contributions will still be sub-dominant in the basis eq. (6.3). The consequences for the iterative procedure in the d.s.l. are the same like for the merging cuts, where in eqs. $(7.12)$ and $(7.13)$ the index 2 has to be replaced by 4 .

An even different kind of continuum limit can be found, when the process of merging and shrinking is put together. In this limit the zero mode contributions in the basis will no longer be sub-dominant. Starting with the following parametrisation,

$$
\begin{aligned}
x_{2} & =x_{3}^{c}+a \nu, \quad \nu>0, \\
x_{3} & =x_{3}^{c} \\
x_{4} & =x_{3}^{c}-a \mu, \quad \mu>0, \\
p & =x_{3}^{c}+a \pi,
\end{aligned}
$$

the modulus and also the ratio $\frac{E(k)}{K(k)}$ will stay finite,

$$
k^{2}=\frac{\nu}{\nu+\mu}+\mathcal{O}(a) .
$$


The modulus may thus reach any value in $(0,1)$. For the special choice $\nu=\mu$, it holds that $k^{2}=k^{\prime 2}=\frac{1}{2}$. The elliptic integrals can then be expressed by the gamma function. Using Legendre's relation, which then reads

$$
2 E(k) K(k)-K(k)^{2}=\frac{\pi}{2},
$$

and the special value $K\left(\frac{1}{\sqrt{2}}\right)=\frac{1}{4 \sqrt{\pi}}\left(\Gamma\left(\frac{1}{4}\right)\right)^{2}$, the ratio of the integrals is given by

$$
\frac{E\left(\frac{1}{\sqrt{2}}\right)}{K\left(\frac{1}{\sqrt{2}}\right)}=\frac{1}{2}+4 \pi^{2} \frac{1}{\left(\Gamma\left(\frac{1}{4}\right)\right)^{4}} .
$$

The $\alpha_{i}, i=2,3,4$, will therefore scale in the desired way,

$$
\begin{aligned}
& \alpha_{1}=\frac{1}{x_{1}-x_{3}^{c}}, \\
& \alpha_{2}=\frac{1}{a \nu}\left(1-\frac{E(k)}{K(k)}\right), \\
& \alpha_{3}=\frac{1}{a \nu}\left(-1+\frac{\nu+\mu}{\mu} \frac{E(k)}{K(k)}\right), \\
& \alpha_{4}=-\frac{1}{a \mu} \frac{E(k)}{K(k)}, \quad k^{2}=\frac{\nu}{\nu+\mu},
\end{aligned}
$$

leaving the form of eq. (6.3) unchanged for $i=2,3,4$. In this kind of d.s.l. the iteration merely simplifies, in contrast to the generic case. The only terms to be left out in eq. (5.20) are those with an index 1.

This ends the short survey of all possible continuum limits to be performed in the two-cut solution.

\section{Conclusion and outlook}

The results presented here complete the perturbative analysis in $\frac{1}{N}$ of the hermitian one-matrix model. All possible solutions classified by the number of cuts are derived in an iterative scheme for higher genus contributions to the loop correlators, which generalises the results of [2] for the one-cut solution. Explicit expressions were given for the one-loop correlator of genus zero and one and for the planar two-loop correlator at different arguments. The latter is universal for each number of cuts $s$, providing a whole set of new universality classes.

The two-cut solution was displayed in full detail, including the genus one contribution to the free energy. When investigating the double-scaling limit, 
the two-cut solution was proven to be equivalent to the one-cut solution in the generic case. However, when taking the continuum limit at merging or shrinking cuts a different behaviour was revealed. A candidate for this new continuum behaviour is still missing.

Up to now in most investigations of matrix models there has been assumed a one-cut structure of the corresponding quantities. The results given here should make it possible to deal with multi-cut structures also in other models, where the loop equation techniques have been applied successfully. Just to name a few examples like the $\mathrm{O}(\mathrm{n})$-model [18] or the supereigenvalue model [19], particularly in the complex matrix model [20] most explicit results should be accessible, completing the analysis of [21]. The hope is beside finding new universality classes and continuum limits there as well the appearance of new types of critical behaviour, which might still be hidden in the full space of solutions.

Acknowledgements: I would like to thank the Niels Bohr Institute for financial support and its warm hospitality, where part of this work was being done. In particular I wish to thank J. Ambjørn, C. Kristjansen and Yu. Makeenko for many valuable hints and discussions. 


\section{A $\frac{d}{d V}(p)$ of the boundary conditions}

While taking the derivative $\frac{d}{d V}(p)$ of the first type of boundary conditions eq. (4.9) is straight forward, the derivative of the second type eq. (4.10)

$$
0=\int_{x_{2 j+1}}^{x_{2 j}} d \lambda M(\lambda) \sqrt{\prod_{i=1}^{2 s}\left(\lambda-x_{i}\right)}, j=1, \ldots, s-1,
$$

is more sophisticated. When applying $\frac{d}{d V}(p)$ to $M(\lambda)$ in the integrand, the resulting function will no longer be analytic, having an extra pole at $p$. Therefore two things have to be taken into account. First, the contour in the expression for $M(\lambda)$ eq. (4.6) cannot be deformed to infinity but in the end of the calculation 8 , taking it to enclose the poles of the integrand only,

$$
M(\lambda)=\oint_{\mathcal{C} \cup \mathcal{C}_{\lambda}} \frac{d \omega}{2 \pi i} \frac{V^{\prime}(\omega)}{(\omega-\lambda) \sqrt{\prod_{i=1}^{2 s}\left(\omega-x_{i}\right)}} .
$$

Second, when applying $\frac{d}{d V}(p)$ to eq. (A.1) for fixed $j$, the argument $p$ must be excluded to be in the interval of integration, $p \notin\left[x_{2 j+1}, x_{2 j}\right]$. Otherwise the real integral between the cuts does not exist, due to eq. (5.12). The final result will have no poles between any of the cuts, allowing for an analytic continuation of $\frac{d x_{i}}{d V}(p)$ to $p$ also being between the cuts.

Taking this into account the derivative $\frac{d}{d V}(p)$ in the form of eq. (5.11) to eq. (A.1) yields

$$
\begin{aligned}
0= & \int_{x_{2 j+1}}^{x_{2 j}} d \lambda \oint_{\mathcal{C} \cup \mathcal{C}_{\lambda}} \\
& \frac{d \omega}{2 \pi i}\left(\frac{1}{(p-\lambda)^{2}(\omega-\lambda)}\right. \\
& \left.\quad+\frac{1}{2} \sum_{i=1}^{2 s} \frac{d x_{i}}{d V}(p) \frac{V^{\prime}(\omega)}{\omega-\lambda}\left(\frac{1}{\omega-x_{i}}-\frac{1}{\lambda-x_{i}}\right)\right) \frac{\phi^{(0)}(\omega)}{\phi^{(0)}(\lambda)} \\
= & \frac{\partial}{\partial p} \int_{x_{2 j+1}}^{x_{2 j}} d \lambda \frac{1}{p-\lambda} \frac{\phi^{(0)}(p)}{\phi^{(0)}(\lambda)}+\frac{1}{2} \sum_{i=1}^{2 s} \frac{d x_{i}}{d V}(p) M_{i}^{(1)} K_{i, j}
\end{aligned}
$$

where now the contours have been deformed back to infinity. The first term

\footnotetext{
${ }^{8}$ Otherwise the extra pole at $p$ would be overlooked.
} 
can be further evaluated, using partial integration,

$$
\begin{aligned}
\frac{\partial}{\partial p} \int_{x_{2 j+1}}^{x_{2 j}} d \lambda \frac{1}{p-\lambda} \frac{\phi^{(0)}(p)}{\phi^{(0)}(\lambda)} & =\frac{1}{2} \int_{x_{2 j+1}}^{x_{2 j}} d \lambda \frac{1}{p-\lambda} \sum_{i=1}^{2 s}\left(\frac{1}{\lambda-x_{i}}-\frac{1}{p-x_{i}}\right) \frac{\phi^{(0)}(p)}{\phi^{(0)}(\lambda)} \\
& =\frac{1}{2} \sum_{i=1}^{2 s} K_{i, j} \phi_{i}^{(1)}(p)
\end{aligned}
$$

which leads to the final form of eq. (5.14).

\section{B The planar two-loop correlator}

The planar two-loop correlator $W_{0}(p, q)$ with $s$ cuts is derived by applying $\frac{d}{d V}(p)$ from eq. (5.11) to the result for $W_{0}(p)$ eq. (4.7),

$$
\begin{aligned}
W_{0}(p, q)= & \frac{1}{2} \frac{1}{(q-p)^{2}} \frac{\phi^{(0)}(q)}{\phi^{(0)}(p)}+\frac{1}{4} \frac{1}{(q-p)} \frac{1}{\phi^{(0)}(p)} \sum_{i=1}^{2 s} \phi_{i}^{(1)}(q) \\
& -\frac{1}{2} \frac{1}{(q-p)^{2}}+\frac{1}{4} \frac{1}{\phi^{(0)}(p)} \sum_{i=1}^{2 s} \frac{1}{p-x_{i}} M_{i}^{(1)} \frac{d x_{i}}{d V}(q) \\
= & \frac{1}{4} \frac{\phi^{(0)}(q)}{\phi^{(0)}(p)}\left(\frac{2}{(q-p)^{2}}+\frac{1}{q-p} \sum_{i=1}^{2 s} \frac{1}{p-x_{i}}+\sum_{i=1}^{2 s} \sum_{l=0}^{s-2} \frac{\alpha_{i, l} q^{l}}{p-x_{i}}\right) \\
& -\frac{1}{2} \frac{1}{(q-p)^{2}},
\end{aligned}
$$

where the result for the $\frac{d x_{i}}{d V}(q)$ eq. (5.15) has been used. Although this is in principle the solution for arbitrary $s$, it would be nice to have it in a form, where the analyticity properties, which follow from its definition, are obviously fulfilled. Namely from eq. (2.6)

$$
W_{0}(p, q)=\frac{d}{d V}(p) W_{0}(q)=\frac{d}{d V}(p) \frac{d}{d V}(q) F_{0},
$$

it is clear, that it must satisfy

- $\operatorname{symmetry} W_{0}(p, q)=W_{0}(q, p)$

- analyticity like $W_{0}(p) \sim \phi^{(0)}(p)$, no higher poles

- asymptotic $\lim _{p \rightarrow \infty} W_{0}(p, q) \sim \mathcal{O}\left(\frac{1}{p^{2}}\right)$ 
- regularity $\lim _{q \rightarrow p} W_{0}(p, q)=W_{0}(p, p)$

- $\left.-\frac{1}{(p-q)^{2}}=\lim _{\epsilon \rightarrow 0}\left(W_{0}(p+i \epsilon, q)\right)+W_{0}(p-i \epsilon, q)\right)$

The last property can be derived from taking the derivative $\frac{d}{d V}(p)$ of eq. (4.3), which may be rephrased as

$$
V^{\prime}(q)=2 \operatorname{Re}\left(W_{0}(q)\right), q \in \sigma .
$$

This last point is clearly satisfied by eq. (B.1), whereas the others are not obvious. In the case of the two-cut solutions however, where the $\alpha_{i, 0}$ are explicitly known, $W_{0}(p, q)$ can be cast into a form, where all points are seen to be true immediately. Inserting eq. (6.4) into eq. (B.1) and using the identity

$$
\begin{aligned}
0 & =2 \prod_{i=1}^{4}\left(p-x_{i}\right)+(q-p)^{2}\left(\left(p-x_{2}\right)\left(p-x_{3}\right)+\left(p-x_{1}\right)\left(p-x_{4}\right)\right) \\
& +(q-p) \sum_{i=1}^{4} \prod_{j \neq i}\left(p-x_{j}\right)-\left(p-x_{1}\right)\left(p-x_{4}\right)\left(q-x_{2}\right)\left(q-x_{3}\right) \\
& -\left(q-x_{1}\right)\left(q-x_{4}\right)\left(p-x_{2}\right)\left(p-x_{3}\right)
\end{aligned}
$$

leads to the planar two-loop correlator with two cuts,

$$
\begin{aligned}
W_{0}(p, q)= & \frac{1}{4(p-q)^{2}}\left(\sqrt{\frac{\left(p-x_{1}\right)\left(p-x_{4}\right)\left(q-x_{2}\right)\left(q-x_{3}\right)}{\left(p-x_{2}\right)\left(p-x_{3}\right)\left(q-x_{1}\right)\left(q-x_{4}\right)}}\right. \\
& \left.+\sqrt{\frac{\left(p-x_{2}\right)\left(p-x_{3}\right)\left(q-x_{1}\right)\left(q-x_{4}\right)}{\left(p-x_{1}\right)\left(p-x_{4}\right)\left(q-x_{2}\right)\left(q-x_{3}\right)}}\right) \\
+ & \frac{1}{4} \frac{1}{\sqrt{\prod_{j=1}^{4}\left(p-x_{j}\right)\left(q-x_{j}\right)}} \frac{E(k)}{K(k)} f\left(\left\{x_{i}\right\}\right)-\frac{1}{2(p-q)^{2}}
\end{aligned}
$$

with

$$
\begin{aligned}
f\left(\left\{x_{i}\right\}\right) & =\frac{x_{2} x_{3} x_{4}\left(x_{2}-x_{4}\right)}{\left(x_{1}-x_{4}\right)\left(x_{2}-x_{1}\right)}+\frac{x_{1} x_{3} x_{4}\left(x_{1}-x_{3}\right)}{\left(x_{1}-x_{2}\right)\left(x_{2}-x_{3}\right)} \\
& +\frac{x_{1} x_{2} x_{4}\left(x_{4}-x_{2}\right)}{\left(x_{2}-x_{3}\right)\left(x_{3}-x_{4}\right)}+\frac{x_{1} x_{2} x_{3}\left(x_{1}-x_{3}\right)}{\left(x_{1}-x_{4}\right)\left(x_{3}-x_{4}\right)} .
\end{aligned}
$$

The same can be done for coinciding arguments, using eq. (5.20), which reads

$$
W_{0}(p, p)=\frac{1}{16} \sum_{i=1}^{4} \frac{1}{\left(p-x_{i}\right)^{2}}-\frac{1}{8} \sum_{i<j}^{4} \frac{1}{\left(p-x_{i}\right)\left(p-x_{j}\right)}+\frac{1}{4} \sum_{i=1}^{2 s} \frac{\alpha_{i}}{p-x_{i}} .
$$


Taking the solution for the $\alpha_{i}$ eq. (6.4) it can be shown, that

$$
\begin{aligned}
\frac{1}{4} \sum_{i=1}^{2 s} \frac{\alpha_{i}}{p-x_{i}} & =\frac{1}{4}\left(\frac{1}{\left(p-x_{1}\right)\left(p-x_{4}\right)}+\frac{1}{\left(p-x_{2}\right)\left(p-x_{3}\right)}\right) \\
& +\frac{1}{4} \frac{1}{\prod_{j=1}^{4}\left(p-x_{j}\right)} \frac{E(k)}{K(k)} f\left(\left\{x_{i}\right\}\right),
\end{aligned}
$$

which leads to the final form of eq. (6.6). It can be checked now, that taking the limit $q \rightarrow p$ of eq. (B.5) will give back the regular expression $W_{0}(p, p)$ as it should.

\section{Elliptic integrals}

The elliptic integrals defined in eq. 6.2) can be expressed by elementary functions and three fundamental integrals, namely the complete elliptic integrals of the first, second and third kind

$$
\begin{aligned}
K(k) & \equiv \int_{0}^{1} d t \frac{1}{\sqrt{\left(1-t^{2}\right)\left(1-k^{2} t^{2}\right)}}, \quad E(k) \equiv \int_{0}^{1} d t \sqrt{\frac{1-k^{2} t^{2}}{1-t^{2}}} \\
\Pi\left(\alpha^{2}, k\right) & \equiv \int_{0}^{1} d t \frac{1}{\left(1-\alpha^{2} t^{2}\right) \sqrt{\left(1-t^{2}\right)\left(1-k^{2} t^{2}\right)}} .
\end{aligned}
$$

With the help of the tables in [17] the $K_{i}, i=1, \ldots, 4$, are then given by

$$
\begin{aligned}
K_{1} \equiv & -\int_{x_{3}}^{x_{2}} \sqrt{\frac{\left(x_{2}-\lambda\right)\left(\lambda-x_{3}\right)\left(\lambda-x_{4}\right)}{x_{1}-\lambda}} \\
= & X\left[\left(-\alpha^{6}+2 \alpha^{4}-2 \alpha^{4} k^{2}+\alpha^{2} k^{2}\right) E(k)-\left(k^{2}-\alpha^{2}\right)\left(\alpha^{4}-2 \alpha^{2}+k^{2}\right) K(k)\right. \\
& \left.+\left(\alpha^{8}-4 \alpha^{6} k^{2}+6 \alpha^{4} k^{2}-4 \alpha^{2} k^{2}+k^{4}\right) \Pi\left(\alpha^{2}, k\right)\right], \\
K_{2} \equiv & -\int_{x_{3}}^{x_{2}} \sqrt{\frac{\left(x_{1}-\lambda\right)\left(\lambda-x_{3}\right)\left(\lambda-x_{4}\right)}{x_{2}-\lambda}} \\
= & X\left[\left(-\alpha^{6}-2 \alpha^{4}+2 \alpha^{4} k^{2}+\alpha^{2} k^{2}\right) E(k)+\left(-\alpha^{4}-2 \alpha^{2}+4 \alpha^{2} k^{2}-k^{2}\right) .\right. \\
& \left.\cdot\left(k^{2}-\alpha^{2}\right) K(k)+\left(\alpha^{8}-4 \alpha^{6}+6 \alpha^{4} k^{2}-4 \alpha^{2} k^{4}+k^{4}\right) \Pi\left(\alpha^{2}, k\right)\right],
\end{aligned}
$$




$$
\begin{aligned}
K_{3} \equiv & \int_{x_{3}}^{x_{2}} \sqrt{\frac{\left(x_{1}-\lambda\right)\left(x_{2}-\lambda\right)\left(\lambda-x_{4}\right)}{\lambda-x_{3}}} \\
= & X\left[\left(3 \alpha^{6}-2 \alpha^{4}-2 \alpha^{4} k^{2}+\alpha^{2} k^{2}\right) E(k)+\left(k^{2}-\alpha^{2}\right)\left(3 \alpha^{4}-2 \alpha^{2}-k^{2}\right) K(k)\right. \\
& \left.+\left(-3 \alpha^{8}+4 \alpha^{6}+4 \alpha^{6} k^{2}-6 \alpha^{4} k^{2}+k^{4}\right) \Pi\left(\alpha^{2}, k\right)\right], \\
K_{4} \equiv & \int_{x_{3}}^{x_{2}} \sqrt{\frac{\left(x_{1}-\lambda\right)\left(x_{2}-\lambda\right)\left(\lambda-x_{3}\right)}{\lambda-x_{4}}} \\
= & X\left[\left(-\alpha^{6}+2 \alpha^{4}+2 \alpha^{4} k^{2}-3 \alpha^{2} k^{2}\right) E(k)+\left(-\alpha^{4}+2 \alpha^{2}-4 \alpha^{2} k^{2}+3 k^{2}\right) .\right. \\
& \left.\cdot\left(k^{2}-\alpha^{2}\right) K(k)+\left(\alpha^{8}-6 \alpha^{4} k^{2}+4 \alpha^{2} k^{2}+4 \alpha^{2} k^{4}-3 k^{4}\right) \Pi\left(\alpha^{2}, k\right)\right]
\end{aligned}
$$

where

$$
\begin{aligned}
X & \equiv-\frac{1}{4} \frac{\left(x_{1}-x_{3}\right)^{\frac{3}{2}}\left(x_{2}-x_{4}\right)^{\frac{7}{2}}}{\left(x_{2}-x_{3}\right)^{2}\left(x_{3}-x_{4}\right)} \\
k^{2} & \equiv \frac{\left(x_{1}-x_{4}\right)\left(x_{2}-x_{3}\right)}{\left(x_{1}-x_{3}\right)\left(x_{2}-x_{4}\right)}, \quad \alpha^{2} \equiv \frac{x_{2}-x_{3}}{x_{2}-x_{4}}
\end{aligned}
$$

The composition into $K(k), E(k)$ and $\Pi\left(\alpha^{2}, k\right)$ is not unique because of possible transformations of the modulus $k$ and the parameter $\alpha$. Using the explicit form of eq. C.2 , the coefficients $\alpha_{i}$ in the solution for the $\frac{d x_{i}}{d V}(p)$ eq. (6.3) can be shown to have the simple form, that is given in eq. (6.4). 


\section{References}

[1] J. Jurkiewicz, M. A. Nowak and I. Zahed, Dirac spectrum in QCD and quark masses, hep-ph/9603308, 1996

[2] J. Ambjørn, L. Chekhov, C. F. Kristjansen and Yu. Makeenko, Matrix model calculation beyond the spherical limit, Nucl. Phys. B404 (1993) 127

[3] O. Lechtenfeld, Eigenvalue tunneling in matrix models, Int. J. Mod. Phys A7 (1992) 2335

[4] J. Jurkiewicz, Chaotic behavior in one-matrix models, Phys. Lett. B261 (1991) 260

[5] M. Sasaki and H. Suzuki, Matrix realization of random surfaces, Phys. Rev. D43 (1991) 4015

[6] D. Sénéchal, Chaos in the hermitian one-matrix model, Int. J. Mod. Phys A7 (1992) 1491

[7] R. C. Brower, N. Deo, S. Jain and C. Tan, Symmetry breaking in the double-well hermitian matrix model, Nucl. Phys. B405 (1993) 166

[8] G. Akemann, Phasenübergänge in Matrixmodellen, $\mathrm{PhD}$ thesis, Universität Hannover, 1996

[9] K. Demeterfi, N. Deo, S. Jain and C. Tan, Multiband structure and critical behaviour of matrix models, Phys. Rev. D42 (1990) 4105

[10] Č. Crnković and G. Moore, Multicritical multi-cut matrix models, Phys. Lett. B257 (1991) 322

[11] Č. Crnković, M. Douglas and G. Moore, Loop equations and the topological phase of multi-cut matrix models, Int. J. Mod. Phys A7 (1992) 7693

[12] O. Lechtenfeld, R. Ray and A. Ray, Phase diagram and orthogonal polynomials in multiple-well matrix models, Int. J. Mod. Phys A6 (1991) 4491

[13] J. Ambjørn, Quantisation of geometry, Lectures given at Les Houches Summerschool on Fluctuating Geometry and Statistical Mechanics, France, Les Houches, session LXII 1994 
[14] F. David, Phases of the large-N matrix model and non-perturbative effects in 2D gravity, Nucl. Phys. B348 (1991) 507

[15] J. Jurkiewicz, Regularisation of one-matrix models, Phys. Lett. B245 (1990) 178

[16] G. Cicuta, L. Molinari and E. Montaldi, Large N phase transitions in low dimensions, Mod. Phys. Lett. A1 (1986) 125

[17] P. F. Byrd and M. D. Friedman, Handbook of Elliptic Integrals for Engeneers and Scientists, Springer Verlag, New York 1971

[18] B. Eynard and C. Kristjansen, Exact solution of the $\mathrm{O}(\mathrm{n})$ model on a random lattice, Nucl. Phys. B455 (1995) 577

[19] J.C. Plefka, Iterative solution of the supereigenvalue model, Nucl. Phys. B444 (1995) 333, The supereigenvalue model in the double-scaling limit, Nucl. Phys. B448 (1995) 355

[20] J. Ambjørn, C. F. Kristjansen and Yu. Makeenko, Higher genus correlators for the complex matrix model, Mod. Phys. Lett. A7 (1992) 3187

[21] G. Akemann, Loop equations for multi-cut matrix models, ITP-UH-1195, DESY 95-066, hep-th/9503185 